\title{
Diagnosis of the Coenosia pedella-group (Diptera: Muscidae), with descriptions of three new species from China
}

\author{
Ping Wang \& Wan-Qi Xue*
}

\begin{abstract}
Wang, P. \& Xue, W. Q. 2015: Diagnosis of the Coenosia pedella-group (Diptera: Muscidae), with descriptions of three new species from China. — Entomol. Fennica 26: 101-109.

The Coenosia pedella-group is established and defined by the combination of the following characters: most species have one presutural dorsocentral seta, costa reaching the tip of media vein, abdomen with distinct spots, hind tibia with dorsal setae, lower calypter at least subequal in length to upper calypter, basis of cerci and surstyli of male short, length of cerci at most four times as long as its width, most of cerci enlarged in distal $1 / 3$ and surstyli subtriangular with width of basal part longer than $1 / 3$ of length. Three new species are described: $C$. disclambosa Xue \& Wang sp. n., C. maoershanensis Xue \& Wang sp. n. and C. sublacteipennis Xue \& Wang sp. n. A key to the males of Palaearctic Region species of the Coenosia pedella-group is given.

P. Wang \& W. Q. Xue, Institute of Entomology, Shenyang Normal University, Shenyang 110034, P. R. China, *Corresponding author'se-mail: xwqfly@sina. com
\end{abstract}

Received 23 May 2014, accepted 5 January 2015

\section{Introduction}

Meigen (1826) described the genus Coenosia for 28 species, and Musca tigrina Fabricius, 1775 was subsequently designated as the type species. It is distributed in all zoogeographic regions of the world and comprises about 699 scientific names, about 151 Coenosia species in the Palaearctic Region and about 105 Coenosia species in China.

The study of the genus Coenosia was confused earlier. In addition to Allognota Pokorny, 1893, there were 17 junior synonyms of the genus Coenosia which Pont $(1977,1986)$ had pointed out in the catalogues of each zoogeographical region. Hennig (1961) revised the Palaearctic species of Coenosia and divided the genus into tigrina-group, albatella-group, tricolor-group, intermedia-group and pumila-group. The pumila-group was divided into pumila-subgroup (including seven species), perpusilla-subgroup (including six species), mollicula-subgroup (including ten species) and lineatipes-subgroup (including 11 species) (Hennig 1961). On the basis of cerci and surstyli of male being short, the length of cerci at most four times as long as its width, most of cerci enlarged in distal $1 / 3$, surstyli subtriangular, and the width of basal part longer than $1 / 3$ of length, we remove Coenosia pedella from the pumila-subgroup.

In the present paper, we establish the Coenosia pedella-group according to the species which have the above characters. The total of 35 species of the Coenosia pedella-group from the Palaearctic Region is included, including three new species described herein. 


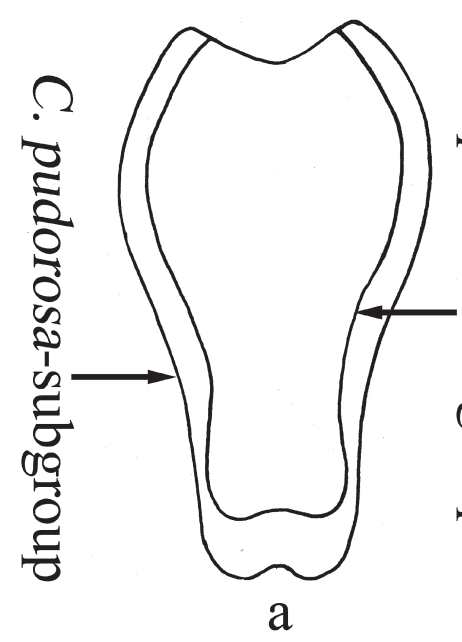

\section{Material and methods}

The specimens examined for this paper were collected by sweeping from brushwood with hand nets. The specimens were examined using a compound light microscope for the external morphological features. For the dissection of terminalia, the end of abdomen was removed and boiled in $10 \% \mathrm{NaOH}$ solution for $15-20$ seconds. After the dissection and study, the abdomen and terminalia were washed and then stored in microvials of glycerine pinned directly underneath the specimens. Illustrations were done in ink and processed using Adobe Photoshop CS.

The type specimens of the three new species are deposited in the Institute of Entomology, Shenyang Normal University, China.

The morphological terminology follows that of McAlpine (1981). Absolute measurements for the body length are given in millimetres. Abbreviations are as follows:

- $f r$ : frontal setae

- acr: acrostichal setae

- $d c$ : dorsocentral setae

- pra: prealar setae

- ial: intra-alar setae

- $a v$ : anteroventral setae

- $a d$ : anterodorsal setae

- $p d$ : posterodorsal setae

- $p v$ : posteroventral setae

- $a$ : anterior setae

- $d$ : dorsal setae

- $p$ : posterior setae
- $\mathrm{r}-\mathrm{m}$ : radial-medial crossvein

- M: media vein

$-\mathrm{R}_{4+5}$ : branch of radius

- dm-cu: discal medial-cubital

- millimeter: $\mathrm{mm}$

\section{Diagnosis of the Coenosia pedella-group (Fig. 1)}

The Coenosia pedella-group is defined by the combination of the following characters: in most species presutural $d c 1$, costa reaching the tip of $\mathrm{M}$, abdomen with distinct spots, hind tibia with $d$, lower calypter at least subequal in length to upper calypter; basis of cerci and surstyli of male short; length of cerci at most four times as long as its width; most of cerci enlarged in distal $1 / 3$, surstyli subtriangular, width of basal part longer than $1 / 3$ of length.

The $C$. pedella-group is divided into two subgroups: C. pedella-subgroup and C. pudorosasubgroup. The surstyli of $C$. pedella-subgroup are bent forwards, while the surstyli of C. pudorosa-subgroup are bent backwards.

\section{Descriptions of new species}

\subsection{Coenosia disclambosa Xue \& Wang sp. n. (Fig. 2)}

Type material. Holotype, male, P.R. China: Pianma town, Lushui county, Yunnan Province, 
a

Fig. 2. Coenosia disclambosa Xue \& Wang sp. n., male. - a. Dorsal view of abdomen. - b. Sternite 5 in ventral view. - c. Posterior view of cerci. $-\mathrm{d}$. Terminalia in profile. scale bars: $0.5 \mathrm{~mm}$ in a), $0.2 \mathrm{~mm}$ in b-d).

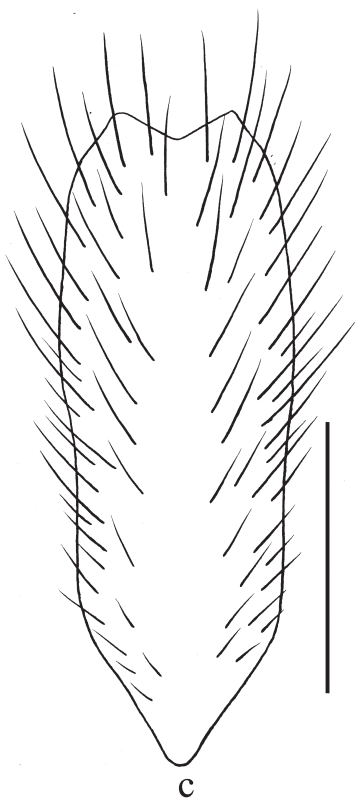

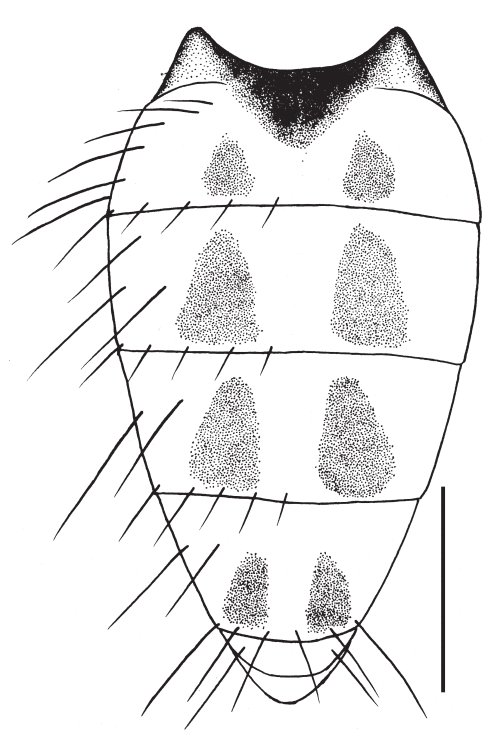

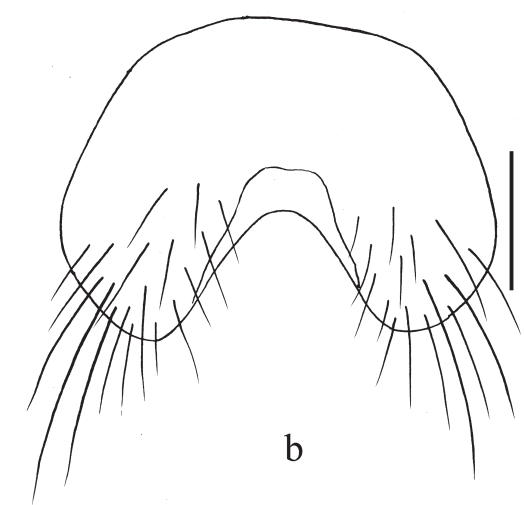

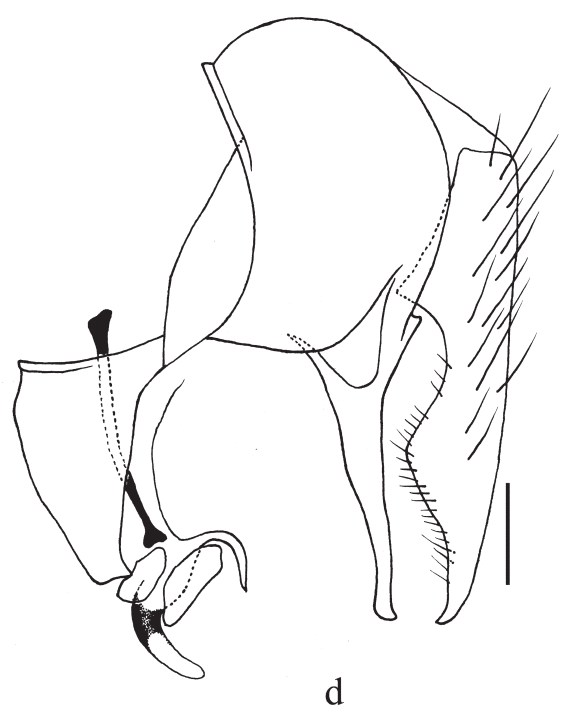

(26.04 N, $\left.98.51^{\circ} \mathrm{E} ; 3,153 \mathrm{~m}\right), 8$. VII.2009, Shu C. Bai leg. Paratypes, 2 males, same data as holotype.

Description. Male. Body length 3.4-3.6 mm.

Head. Eye bare, lower posterior margin not concave; frons about one-third of head width; frontal vitta dark brown, sparsely covered with orange-gray pruinosity, about 2.5 times as wide as fronto-orbital plate; lunule dark orange; frontal triangle reaching front of frons; ocellar setae long, longer than frontal width; inner vertical seta about four times as long as outer vertical seta; two pairs of $f r$, a pair of upper orbital setae; fronto-orbital plate with orange-gray pruinosity; parafacial and gena with green-gray pruinosity, parafacial about half width of postpedicel; antenna black, the anterior part of pedicel with light gray pruinosity spots, postpedicel about 2.2-2.5 times as long as broad, the tip rounded; arista entirely covered with short hairs, the longest hairs as long as basal diameter of arista; epistoma not projecting; frontal angle situated in front of vibrissal an- 
gle; gena about $1 / 5$ of eye in height; genal hairs, and postgenal hairs black; occiput with greengray pruinosity; prementum black and shining, about 2.5 times as long as broad; palpus black, about 1.2 times as long as prementum; labellum about 1.4 times as long as width of prementum.

Thorax. Ground colour black with green-gray pruinosity; scutum with three black vittae, reaching the posterior half of postsutural area of scutum, two rows of hair-like $a c r ; d c 1+3$, posthumeral seta $1+0$, ial $0+2$, without pra; notopleuron bare; both apical and basal scutellar setae developed, lateral surface and ventral surface of scutellum bare; proepisternum and basisternum of prosternum concave in centre; anepimeron, meron and katepimeron bare; proepisternal setae 2, upper proepimeral setae 2; anterior anepisternal seta 1 , both anterior and posterior spiracles small and orange; katepisternal setae placed as an isosceles triangle.

Wings. Hyaline, veins orange; basicosta dark brown; costa reaching tip of $\mathrm{M}$; costal spine subequal in length to $\mathrm{r}-\mathrm{m}$; calypters yellowish, lower calypter about 1.4 times as long as upper one; haltere yellowish.

Legs. Coxae, tarsi, fore femur, distal $2 / 5$ of mid femur and distal $1 / 3$ of hind femur blackbrown, the remaining parts of legs yellow; fore tibia with 1 submedial $p$ which is strong and long, about $2 / 5$ of fore tibia in length; mid femur with 4-5 av in basal half, basal $2 / 3$ with $p v$ row, 2 preapical $p d$; mid tibia with 1 submedial $p d$, without $a d$ and $p v$; hind femur with complete $a v$ row which is irregular, $p v$ row in basal $2 / 5$, about 4-5; hind tibia with 1 submedial $a v, 1$ median $a d, 1$ preapical $d$, without apical $p v$; distal part of tarsi not widened; tarsi longer than tibiae; claws subequal in length to pulvilli, about $3 / 4$ of fifth tarsomere in length.

Abdomen. Long-oval in dorsal view, ground colour black with gray to blue-gray pruinosity; tergites $1+2-5$ each with a pair of large blackbrown lateral spots, without median vitta, posterior marginal setae of each tergite weak, 1-2 pairs of discal setae which are strong; sparsely covered with hairs; sternite 1 bare.

Female. Unknown

Etymology. The species name refers to male characters similar to C. clambosa Wei \& Yang, 2007 and Latin word "dis-" means "un-".
Remarks. This new species resembles $C$. clambosa Wei \& Yang, 2007, but can be distinguished from the latter by the tip of postpedicel rounded; arista entirely covered with short hairs, the longest hairs as long as basal diameter of arista; thorax ground colour black; scutum with three black vittae; costal spine subequal in length to $\mathrm{r}-\mathrm{m}$; coxae and fore femur black-brown; abdomen ground colour black.

\subsection{Coenosia maoershanensis Xue \& Wang sp. n. (Fig. 3)}

Type material. Holotype, male, P.R. China: Mt. Maoer, Guangxi Province, $\left(25.49^{\circ} \mathrm{N}, 111.03^{\circ} \mathrm{E}\right.$; $2,000 \mathrm{~m}), 14$.V.2004, Dong Zhang leg. Paratypes, 7 males and 3 females, same data as holotype.

Description. Male. Body length 5.4-5.6 mm.

Head. Eye bare, lower posterior margin not concave; frons about $0.27-0.30$ of head width; frontal vitta black, with orange-gray pruinosity, about 3.5-4.0 times as wide as fronto-orbital plate; frontal triangle with brown-gray pruinosity, reaching upper $2 / 5$ of frons; ocellar setae large and strong, subequal to the frons width; inner vertical seta about 2.5-2.8 times as long as outer vertical seta; three pairs of $f r$, the middle pair slightly weak; a pair of upper orbital setae; fronto-orbital plate and parafacial with yellowgray pruinosity, gena with grayish pruinosity; parafacial about 2/5-1/2 of postpedicel width; antenna black, anterior apical margin of pedicel with orange colour, with grayish pruinosity, postpedicel about three times as long as broad, anterior lower margin as an obtuse angle; arista entirely covered with short hairs, the longest hairs shorter than basal diameter of arista; the widened part of arista about $1 / 6$ of arista length in basal part; lunule dark brown; epistoma not projecting; frontal angle situated in front of vibrissal angle; gena about $1 / 6$ of eye in height; genal hairs and postgenal hairs black, occiput with green-gray pruinosity; prementum black and shining, about 2.5 times as long as broad; palpus brown to dark brown, subequal in length to prementum; lateral part of labellum with 3-4 pairs of upcurved teeth, oral disc with a pair of broad teeth on posterior part.

Thorax. Ground colour black, lateral surface 
Fig. 3. Coenosia maoershanensis Xue \& Wang sp. n. - a. Male, sternite 5 in ventral view. - b. Male, posterior view of cerci. - c. Male, terminalia in profile. - d. Female, sternites $1-5$ in ventral view. - e. Female, spermatheca. $-\mathrm{f}$. Female, oviscapt in ventral view. - g. Female, oviscapt in dorsal view. Scale bars $0.5 \mathrm{~mm}$, except $0.2 \mathrm{~mm}$ in b), and $0.1 \mathrm{~mm}$ in e).
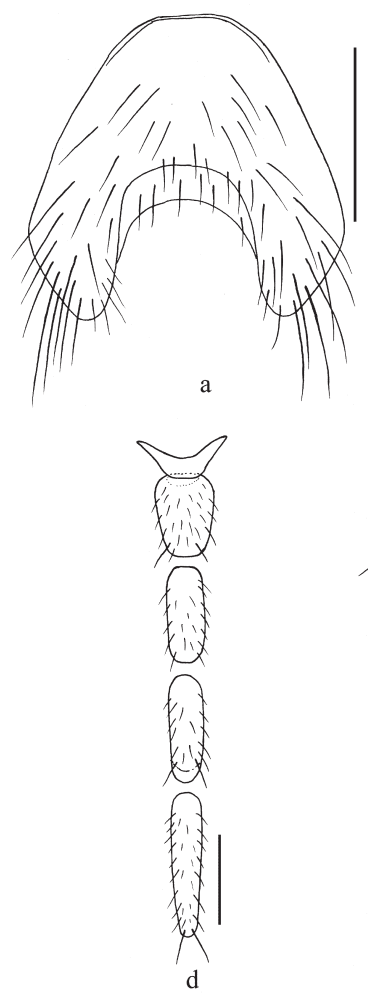

with green-gray pruinosity; scutum with orange pruinosity, along the row of $a c r$ and the row of $d c$ with three brown vittae, median vitta reaching scutoscutellar suture; spacing between the two rows of acr narrow, acr as hairs; $d c 1+3$, ial $0+2$, without pra, posthumeral seta $1+0$; notopleuron bare; both apical and basal scutellar setae developed, lateral surface and ventral surface of scutellum bare; proepisternum and basisternum of prosternum concave in centre; anepimeron, meron and katepimeron bare; proepisternal setae 2 (one of them short); proepimeral setae 2 (the lower one downcurved); anterior anepisternal seta 1; anterior and posterior spiracles small and orange; katepisternal setae placed as an isosceles triangle.

Wings. Hyaline, slender and long, veins orange; basicosta orange-yellow, costal spine distinct; costa reaching the tip of $\mathrm{M} ; \mathrm{R}_{4+5}$ and $\mathrm{M}$ straight, nearly parallel; dm-cu straight; calypters yellowish, lower calypter projecting, about 1.8 times as long as upper one; haltere yellowish.
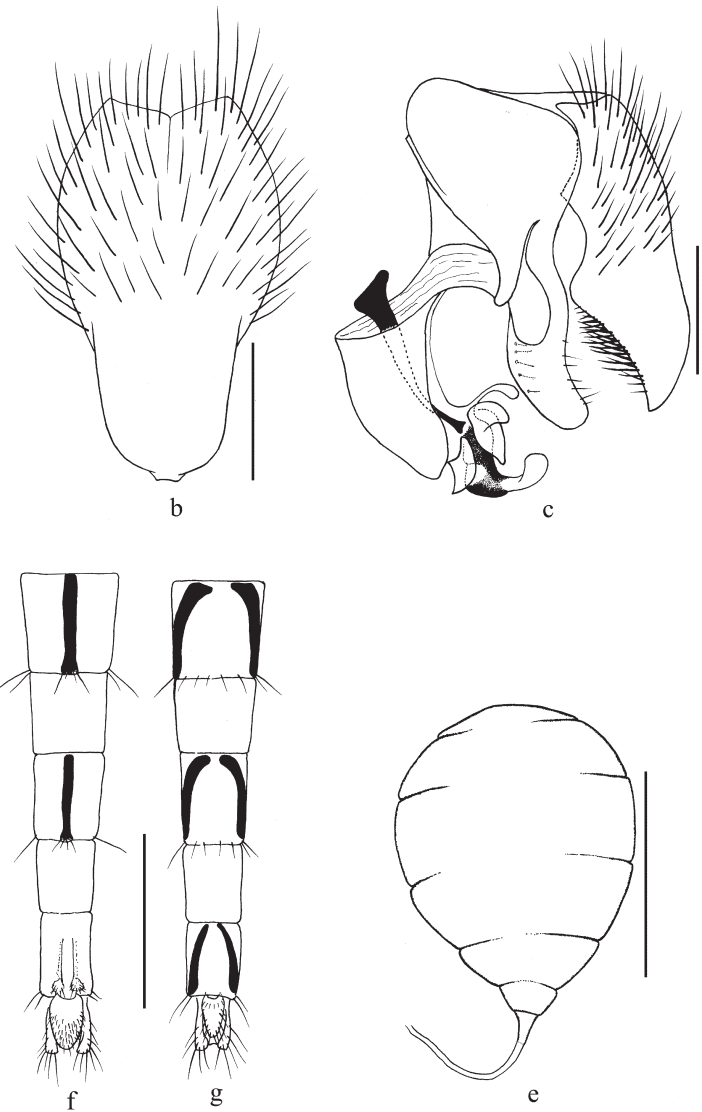

Legs. Trochanters and tibiae yellow, distal $2 / 3-4 / 5$ of mid and hind tibiae orange or orangeyellow, basal and distal parts of fore femur with a little yellow colour, basal $1 / 6$ of mid femur yellow, basal 1/5-1/4 of hind femur yellow, the remaining parts of legs dark black; fore tibia with 1 submedial $p$ which is thin, without $a d$; mid femur with a row of $a v, 3$ strong $p v$ in basal half; mid tibia without submedial ad (one specimen with 1 ad on one side), 1 median $p d$; hind femur sparsely with an irregular row of $a v$ and $p v$, preapical, submedial and supermedial $a v$ long and large, subbasal, submedial, supermedial, preapical $p v$ long and large; hind tibia with 1 submedial $a v, 1$ submedial $\mathrm{ad}$, as long as 2/7 of tibia in length, 1 preapical $d$, without $p d, p$ and apical $p v$; apical part of tarsi not widened; tarsi slightly longer than tibiae; claws longer than pulvilli, claws about $4 / 5$ of fifth tarsomere in length.

Abdomen. Ground colour black, slender, the dorsal part slightly bent, with green-gray pruinosity; tergites $1+2-5$ each with a pair of brown 


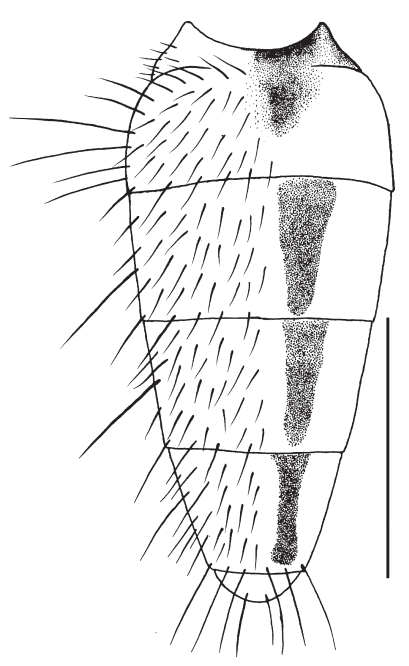

a

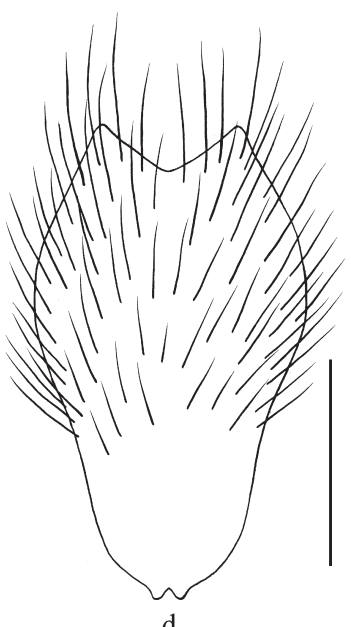

d
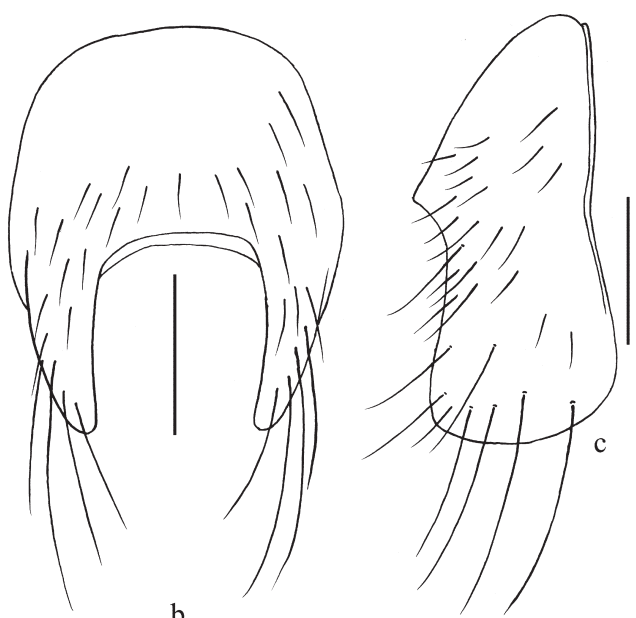

b

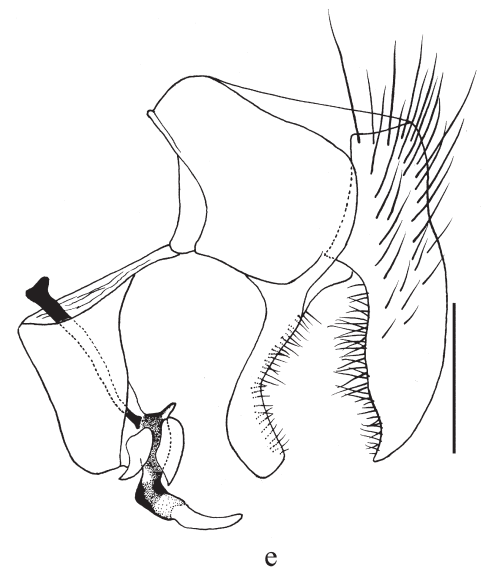

Fig. 4. Coenosia sublacteipennis Xue \& Wang, sp. n., male. - a. Dorsal view of abdomen. - b. Sternite 5 in ventral view. $-c$. Sternite 5 in profile. - d. Posterior view of cerci. - e. Terminalia in profile. Scale bars: $1 \mathrm{~mm}$ in a), $0.2 \mathrm{~mm}$ in b-e). lateral spots, discal setae slightly strong, the row of posterior marginal setae incomplete; sternite 1 bare, sternites 2-4 slender, posterior margin of sternites 2-4 each with a pair of setae, sternite 5 dark orange, lateral lobes slightly broad.

Female. Body length 5.6-5.8 mm. Ocellar setae slightly longer than frontal width; mid tibia with 1 submedial $a d$; abdomen oval, lateral spots of tergites 3-5 not as distinct in male; other characters as male.

Etymology. The species name refers to its type locality.

Remarks. This new species resembles $C$. grandis Xue \& Zhao, 1998, but can be distinguished from the latter by frontal triangle reaching upper 2/5 of frons; ocellar setae large and strong, subequal to frons width; anterior lower margin of postpedicel as an obtuse angle; arista entirely covered with short hairs; gena about $1 / 6$ of eye in height; costal spine distinct; tergites $1+2-5$ each with a pair of brown lateral spots.

\subsection{Coenosia sublacteipennis Xue \& Wang, sp. n. (Fig. 4)}

Type material. Holotype, male, P.R. China: Mt. Maoer, Guangxi Province, $\left(25.49^{\circ} \mathrm{N}, 111.03^{\circ} \mathrm{E}\right.$; 350-2,100 m), 29.IV.-4.V.2009, Chun T. Zhang leg. Paratypes, 2 males, same data as holotype.

Description. Male. Body length 4.5-4.7 mm.

Head. Eye bare, lower posterior margin not concave; lateral margins of frons parallel, about $0.40-0.42$ of head width; frontal vitta dark black, 
with dark gray pruinosity, about 2.8-3.0 times as wide as fronto-orbital plate; frontal triangle with light orange pruinosity, reaching lower $3 / 4$ of frons; ocellar setae shorter than width of frons; inner vertical seta about 2.0-2.4 times as long as outer vertical seta; three pairs of $f r$, the middle pair slightly weak; a pair of upper orbital setae; fronto-orbital plate, parafacial and gena with grayish pruinosity; parafacial about half of postpedicel width; antenna black, postpedicel about 3.5 times as long as broad; arista entirely covered with short hairs, the longest hairs as long as basal diameter of arista; distance between apical part of antenna and epistoma about $1 / 3$ of postpedicel length; anterior part of pedicel with grayish pruinosity, apical margin yellow; epistoma not projecting; frontal angle situated in front of vibrissal angle; gena about $1 / 7$ of eye in height; genal hairs and postgenal hairs black, occiput with green-gray pruinosity; prementum black and shining, about three times as long as broad; palpus black, slightly shorter than prementum; labellum small, about 1.3 times as long as width of prementum.

Thorax. Ground colour black, lateral surface with green-gray pruinosity; middle part of scutum with orange-gray pruinosity, along the row of $a c r$ and the row of $d c$ each with a dark brown vitta, $1-2$ irregular rows of $a c r ; d c 1+3$, ial $0+1$, without pra; notopleuron bare; both basal and apical scutellar setae developed; lateral surface and ventral surface of scutellum bare; proepisternum and basisternum of prosternum concave in centre; anepimeron, meron and katepimeron bare; proepisternal setae 2, the length of them not equal; proepimeral setae 2 (one of them short and decurved), without anterior anepisternal seta; both anterior and posterior spiracles small and brown; katepisternal setae placed as an isosceles triangle.

Wings. Slightly hyaline, veins brown-yellow; basicosta dark brown, tegula yellow; costal spine small; costa reaching the tip of $\mathrm{M} ; \mathrm{R}_{4+5}$ and $\mathrm{M}$ straight, parallel; calypters yellowish, lower calypter slender, about 1.8 times as long as upper one; haltere yellowish.

Legs. Tarsi and distal $1 / 4$ of femora in dorsal surface (distal $3 / 5$ of fore femur) brown, the remaining parts of legs yellow; fore tibia with 1 submedial $p v, 1$ preapical $d$; mid femur with $2-3$ $a v$ in basal $1 / 3,2$ preapical $p d, 3-4 p v$ in basal 2/3; mid tibia with 1 submedial $p$, without $a d$; hind femur with $4 a v$ (the subbasal one small), spacing between them equal, middle part with $3 p v, 1$ preapical $p d$; hind tibia with 1 submedial $a v, 1$ median $a d, 1$ preapical $d$, without $p d$ and apical $p v$; tarsi slightly longer than tibiae; claws subequal in length to pulvilli, claw of fore leg long, about $4 / 5$ of fifth tarsomere in length, claws of mid and hind legs about $3 / 5$ of fifth tarsomere in length.

Abdomen. Ground colour black; long-conical in dorsal view, with dark to green-gray pruinosity; sparsely covered with hairs; posterior marginal setae of tergites $1+2-4$ weak, each with a distinct lateral seta; tergites $1+2-5$ each with a pair of bar dark brown spots, tergite 6 and sternite 1 without hairs, sternites 2-4 rectangular, lateral lobes of sternite 5 broad, distal part of lateral lobes with 3-5 long setae.

Female. Unknown.

Etymology. The species name refers to male terminalia similar to C. lacteipennis (Zetterstedt, 1845 ) and Latin word "sub" means "similar".

Remarks. This new species resembles $C$. lacteipennis (Zetterstedt, 1845), but can be distinguished from the latter by parafacial about half of postpedicel width; postpedicel about 3.5 times as long as broad; dc $1+3$; distal $1 / 4$ of femora in dorsal surface (distal $3 / 5$ of fore femur) brown, the remaining parts of legs yellow; mid tibia without ad.

\section{A key to the males of Palaearctic Region species of the Coenosia pedella-group}

1. $d c 2+3 \quad$ C. lacteipennis (Zetterstedt, 1845)

- $d c 1+3 \quad 2$

2. Costa slightly beyond $R_{4+5} \quad 3$

- Costa reaching the tip of M 4

3. Proepisternal seta 1; mid femur and mid tibia yellow, hind femur and hind tibia blackbrown C.connectens (Hennig, 1961)

- Proepisternal setae 2; mid and hind legs black C. oligochaeta Hennig, 1961

4. Abdomen without spots 5

- Abdomen with distinct spots 8

5. Hind tibia without $d$

C. amplicauda Xue \& Zhu, 2006 
- Hind tibia with $d$

6. Lower calypter at least subequal in length to upper calypter C.parva Xue \& Cui, 2001

- Lower calypter shorter than upper calypter 7

7. Tibiae and first to fourth tarsomeres of fore leg yellow

C. sparagmocerca Zheng et al., 2004

- Distal and basal parts of tibiae yellow, tarsi black C.forcipiungula Xue \& Zhang, 2011

8. Hind tibia without $d$

- Hind tibia with $d$

C. tumidicauda Xue, 2005

9. Lower calypter at least subequal in length to upper calypter

- Lower calypter shorter than upper calypter 10

10. Fore femur with black or black-brown colour

- Fore femur entirely yellow

11. Tibiae yellow C. carinata Cui \& Li, 1996

- Tibiae black C. latisponsa Xue \& Zhang, 2011

12. Mid tibia without $a d$ or $p d$, hind tibia without ad C.flavescentis Wei \& Yang, 2007

- Mid tibia with $a d$ and $p d$, hind tibia with $a d$ C. subflaviseta Xue \& Cui, 2001

13. Lower calypter subequal in length to upper calypter

- Lower calypter distinctly projecting, longer than upper calypter

14. Femora yellow

- Femora black

C. subcarinata Xue \& Zhu, 2008

\section{C. penicullicauda Xue \& Wang, 2008}

15. Proepisternal seta 1

- Proepisternal setae 2

16. Flagellomere 1 with cone-like process at apex

C. grisella Hennig, 1961

- Flagellomere 1 without cone-like process at apex C.xanthocera Hennig, 1961

17. Tibiae black

- Tibiae with yellow colour

19

18. Mid tibia with $1 \mathrm{ad}$, mid femur with a complete row of $p v$

C. appendimembrana Xue \& Zhu, 2008

- Mid tibia without $a d$, mid femur with 2-3pv

C. octosignata Rondani, 1866

19. At least basal parts of mid and hind femora yellow

- Femora entirely dark brown

C. longiquadrata Xue \& Wang, 1992
20. At least basal $2 / 3$ of fore femur dark brown 21

- Basal part of fore femur with yellow colour 26

21. Mid and hind femora yellow

C. monticola Xue \& Zhao, 1998

- At least distal 1/4 of mid and hind femora with dark colour

22. Mid tibia without ad 23

- Mid tibia with ad 24

23. Fore femur entirely black-brown, mid tibia without p C. disclambosa Xue \& Wang sp.n.

- Distal part of fore femur yellow, mid tibia with $\mathrm{p} \quad$ C. clambosa Wei \& Yang, 2007

24. Tarsi black, the remaining parts of legs yellow C. campestris (Robineau-Desvoidy, 1830)

- Legs colour not as above 25

25. Distal half of mid and hind femora black; first tarsomere of fore leg yellow, first tarsomere of other legs dark brown or black

C. verralli Collin, 1953

- Distal 1/4 of mid femur yellow-orange, distal $1 / 3$ of hind femur dark brown; tarsomeres $1-4$ of fore leg and first tarsomere of mid leg yellow C.pygmaea (Zetterstedt, 1845)

26. Antenna black or black-brown 28

- Antenna with yellow or orange colour 27

27 . Basal $1 / 6$ of mid femur yellow, basal $1 / 5-1 / 4$ of hind femur yellow, remaining parts of femora dark black

C. maoershanensis Xue \& Wang sp.n.

- Distal 1/3 of mid and hind femora dark yellow to light brown, remaining parts of femora yellow C. pudorosa Collin, 1953

28. Scutum without spots 33

- Scutum with spots 29

29. Palpus dark yellow

C. nigrifrons Cui \& Li, 1996

- Palpus black or black-brown 30

30. Tarsi brown or black-brown 31

- Tarsi yellow 32

31. Tibiae yellow

C. sublacteipennis Xue \& Wang sp. $\mathbf{n}$.

- Basal 1/4 of tibiae yellow, remaining parts of tibiae light orange to orange

C. grandis Xue \& Zhao, 1998

32. Hind tibia without $p d$

C.paludis Tiensuu, 1939

- Hind tibia with $p d$ C. luteipes Ringdahl, 1930

33. Palpus black or black-brown 34

- Basal half of palpus orange-yellow, distal half dark brown C.mandschurica Hennig, 1961 
34. Apical scutellar seta distinctly shorter than the basal one (basal part of surstyli broad, distal part narrow) C.pedella (Fallén, 1825)

- Apical scutellar seta subequal in length to the basal one (basal part of surstyli narrow, distal part broad)

C. spatuliforceps Xue \& Zhao, 1998

Acknowledgements. This study was supported by the National Nature Science Foundation of China (No. 31172139, 31401990). Special thanks to professor Chuntian Zhang, Shuchong Bai and Dong Zhang for providing the examined materials.

\section{References}

Collin, J. E. 1953: Some additional British Anthomyiidae (Diptera). - Journal of the Society for British Entomology 4: 169-177.

Cui, Y. S. \& Li, L. Z. 1996: Four new species of Coenosia from northeastern China (Diptera: Muscidae). - Entomologia Sinica 3(3): 213-220.

Fabricius, J. C. 1775: Systema entomologiae, sistens insectorvm classes, ordines, genera, species, adiectis synonymis, locis, descriptionibvs, observationibvs. Flensbvrgi et Lipsiae: 1-832.

Fallén, C. F. 1825: Monographia Muscidum Sveciae. Part. IX ultima. — Lundae: 81-94.

Hennig, W. 1961: Muscidae. - In: Lindner, E. (ed.), Die Fliegen der palaearktischen Region, 63b: 518-620. Schweizerbart, Stuttgart. 620 pp.

McAlpine, J. F. 1981: Morphology and terminology adults. - In: McAlpine, J. F., Peterson, B. V., Shewell, G. E., Teskey, H. J., Vockeroth, J. R. \& Wood, D. M. (eds), Manual of Nearctic Diptera, Volume 1, 27: 93. - Agriculture Canada Monograph, 674 pp.

Meigen, J. W. 1826: Systematische beschreibung der bekannten europäischen zweiflügeligen Insekten. Schulz, Hamm. 220 pp.

Pont, A. C. 1977: Family Muscidae. — In: Delfinado, M. D. \& Hardy, D.E. (eds), A Catalog of the Diptera of the Oriental Region. Vol. III: 451-523. — University of Hawaii, Honolulu, 585 pp.

Pont, A. C. 1986: Family Muscidae. — In: Soós, Á. \& Papp, L. (eds), Catalogue of Palaearctic Diptera. Vol. 11: 57-215. - Akadémiai Kiadó, Budapest, 346 pp.

Pokorny, E. 1893: Eine alte und einige neue Gattungen der
Anthomyiden. - Wiener Entomologische Zeitung 12: $53-64$

Ringdahl, O. 1930: Entomologische Ergebnisse der schwedischen Kamtchatka-Expedition 1920-1922. 30. Diptera Brachycera 3. Fam. Muscidae. - Arkiv for Zoologi 21A (20): 1-16.

Robineau-Desvoidy, A. J. B. 1830: Essai sur les Myodaires. - Mémoires Présentés par divers Savants a 1 Académie Royale des Sciences de 1 Institut de France 2: 1813.

Rondani, C. 1866: Anthomyinae Italicae, collectae distinctae et in ordinem dispositae. - Atti della Societ Italiana di Scienze Naturali e del Museo Civico di Storia Naturale di Milano 9: 68-217.

Tiensuu, L. 1939: Beiträge zur Kenntnis der Musciden (Dipt.). Finnlands. 2. - Annales Entomologici Fennici 5: 241-255.

Wei, L. M. \& Yang, Z. H. 2007: Diptera, Muscidae. - In: Li, Z. Z., Yang, M. F. \& Jin, D. C. (eds), Landscape Insects of Mountain Leigongshan: 457-512. — Guizhou Science and Technology Press, 759 pp.

Xue, W. Q. \& Cui, Y. S. 2001: Five new species of Coenosia (Diptera: Muscidae) in Xinjiang China. - Entomologia sinica 8(2): 102-110.

Xue, W. Q., Song, W. H., \& Zheng, L. J. 2005: Three new species of the genus Coenosia from Yunnan Province, China. - Chinese journal of pest control 21(2): 118121.

Xue, W. Q. \& Wang, M. F. 1992: Two new species of the genus Coenosia from China (Diptera: Muscidae). Acta Entomologica Sinica 35(3): 365-368.

Xue, W. Q. \& Zhao, B. G. 1998: Four new species of the genus Coenosia from Zhejiang, China. - Acta Zootaxonomica Sinica 23(3): 319-324.

Xue, W. Q. \& Zhang, X. S. 2011: Geographic distribution of Coenosia Meigen (Diptera: Muscidae) of Yunnan, China, with descriptions of four new species. - Deutsche Entomologische Zeitschrift 58(1): 155-164.

Xue, W. Q. \& Zhu, Y. 2006: Three new species of Coenosia Meigen (Diptera: Muscidae) from P.R. China, with a key to the Chinese species of the genus. - Zootaxa 1326: 1-16.

Xue, W. Q., Zhu, Y., Wang M. F., \& Zhang C. T. 2008: Six new species of Coenosia Meigen (Diptera: Muscidae) from China. - Oriental Insects 42: 143-153.

Zetterstedt, J. W. 1845: Diptera Scandinaviae disposita et descripta (part). — Lundae, 4: 1281-1738.

Zheng, L. J., Xue, W. Q., \& Tong, Y. F. 2004: Five new species of the genus Coenosia (Diptera: Muscidae) from China. - Journal of northeast forestry university 32 (3): 48-51. 